\title{
Can targeted genetic testing offer useful health information to adoptees?
}

\author{
Thomas May, PhD ${ }^{1,2}$, Kimberly A. Strong, $\mathrm{PhD}^{1}$, Muin J. Khoury, MD, $\mathrm{PhD}^{3}$, and \\ James $\mathrm{P}$. Evans, $\mathrm{MD}, \mathrm{PhD}^{4}$
}

In 2004, the US Surgeon General launched the Family History Public Health Initiative to increase awareness and discussions regarding family health history (FHx). FHx reflects the combined influences of shared genetic, behavioral, and environmental factors in families. The collection and use of FHx have already been shown to be useful in risk assessment and preventive intervention for cardiovascular disease, osteoporosis, and cancer surveillance/chemoprevention, to name a few. ${ }^{1-3}$ Yet many segments of the population lack adequate access to the genetic component of FHx that might inform their health management. Here, we focus on adoptees, whose lack of genetic FHx normally reflects separation from biological parents as an inherent feature of adoption. However, our fundamental point is applicable to anyone who lacks access to genetic FHx information, which can occur for many reasons. Lack of genetic FHx is not inconsequential; for example, a recent Swedish study of adoptees emphasizes the importance of genetic factors over environmental factors for several cancers. ${ }^{4}$ Current genetic testing capabilities are at a stage where it is legitimate to ask, "Could targeted genetic analysis offer any potential benefit for those individuals who have no or limited access to family history, such as many adoptees?" And might the potential benefits and risks of genetic analysis differ between adoptees and those with access to their family history information?

According to US Census data (2000), adoptees account for more than $2.5 \%$ of the US population (7.8 million). Worldwide, the United Nations estimates that at least 260,000 children are adopted annually, and the number of children globally who have lost both parents is more than 60 times this number. ${ }^{5}$ Although some adoptees have access to family history information (for example, through open adoption or biological relative adoption), many do not. One study found that for adoptees in general, family medical information was available for less than half of birth fathers. ${ }^{6}$ For international adoptees (currently one-quarter of adoptions), ${ }^{7}$ this problem is exacerbated not only by logistical difficulties but also by well-established problems surrounding the limitations of medical records in many countries from which international adoptions occur. For nearly two-thirds of international adoptees, no written medical records exist, even for basic things such as vaccination status. ${ }^{8,9}$ Moreover, even adoptees with access to some FHx information often lack ongoing, iterative refinement of this knowledge available to biologically related families who have regular, multi-generational interaction.

Clinical actionability of FHx in practice is often seen in situations in which the pattern of disease in the family suggests the presence of a genetic disorder and indicates the need for earlier screening or other interventions. Many adoptees do not have access to this potentially lifesaving information. However, emerging genomic technologies are beginning to offer the possibility of accessing some of this medically actionable genetic information. An important point of similarity between genetic data and family history is that both have the greatest clinical impact on medical care when they serve to indicate an uncommon, but dramatic, risk such as that indicated by a strong family history of early diagnosis of breast cancer, colon cancer, or the like (knowledge of which can lead to recommendations for earlier screening or overtly preventive intervention). It is in several of these areas where the strongest gene-disease associations lie, such as for breast cancers and colorectal cancers. Although genetic analysis is unlikely to provide a replacement for family history when available, if properly targeted and interpreted, such analysis may have the potential to provide useful information regarding health risks when no information currently exists. Given an inability to realize family history-derived health benefits (i.e., a need for earlier screening or interventions) by many adoptees, it is critical to evaluate the potential of carefully considered genetic evaluation in this population.

\section{APPLICATION OF GENETIC TECHNOLOGIES FOR HEALTH RISK IDENTIFICATION}

Despite the potential described, uncertainty exists regarding the ability of genomic analyses of common genetic variants to play a role in the identification of inherited disease risk. Some 
of this surrounds previous studies that, rather predictably, found genome-wide association studies testing to be far less useful than family history alone but of some possible use as a supplement to family history. ${ }^{10,11}$ Also, the most successful applications of genetic testing often involve testing multiple related individuals across several generations, which is something quite difficult to realize in practice. Also problematic is that many genes confer intermediate degrees of increased disease risk (e.g., CHEK2) and that an individual's actual risk remains dependent on a variety of factors, including family history. ${ }^{10}$ Thus, although integrating family history information and genomic data is optimal, not everyone has an accurate and/or complete family history or access to biological relatives who could participate. Moreover, interpretation of the genome is currently limited. The inevitable discovery of incidental findings is one of the most controversial and problematic issues in genomic medicine, and false reassurance from negative reports or overreaction to positives from indeterminate or low-prevalence gene-disease associations (exacerbated by the high number of variants of unknown significance) continue to challenge genetic clinicians.

Given these limitations, the greatest potential for use of genomic information in the absence of family history may be derived from analysis of a limited number of highly penetrant, well-established, and actionable gene-disease associations that confer relatively high levels of risk. As discussed previously, such variants are often most recognizable as "mapping onto" those conditions commonly thought of as identifiable through traditional FHx. The number of genes to be analyzed under such an approach would be small given the currently poor potential for robust prevention of most conditions. However, like newborn screening panels used in many states, such information may prove critically valuable to a subset of individuals. ${ }^{12}$

A number of important issues will need to be addressed to realize the promise of such an approach. For example, it might well be the case that when we identify an individual with the BRCA1 mutation who has no positive family history of disease (in our context, because they lack family health history information), the risk for that person may be somewhat lower than that for an individual with, for example, multiple relatives who had breast cancer. Lack of knowledge about FHx will thus complicate counseling for BRCA-positive individuals (e.g., how do we discuss options like a risk-reducing bilateral mastectomy with a woman who is positive for BRCA1 but has no positive family history indicators?). In summary, for many gene-disease associations, interpretation of risk is partly dependent on contexts that may be different for adoptees lacking access to $\mathrm{FHx}$, and thus may require rethinking how we approach both counseling and risk stratifications within clinical reports.

Nonetheless, for people who do not have access to FHx, a conservative approach consisting of targeted analysis of highly actionable genes to discover mutations that would lead to clear clinical recommendations designed to avert a significant threat to health may be useful.

Several examples of targeted panels that might serve as starting points for FHx information for adoptees have already been proposed. Lists identified by GeneScreen, ${ }^{13}$ the Clinical Sequencing Exploratory Research Consortium, ${ }^{14}$ and the American College of Medical Genetics and Genomics working group on incidental findings ${ }^{15}$ all identify highly penetrant, actionable gene disease associations that have high morbidity and/or mortality. Each of these has its own controversies and limitations, but they represent an excellent starting point to define a subset of targeted gene testing that may be useful to adoptees. The list of genes and conditions identified would likely be modified for adult adoptees due to differing contexts for understanding "actionability" in adult adoptees versus the general population. For example, when the strength of gene-disease association is highly dependent on interpretation in the context of family history, the specific mutation in question might be less appropriate for adoptees than for the general public. In addition, some FHx information is important not for health screening, but for other purposes, e.g., reproduction. For example, TaySachs is perhaps the most well-known hereditary condition for which lack of genetic information (in this case, that of ancestry) might be relevant. The exact list of genes that would be optimal for analysis will depend on a variety of factors, including considerations of penetrance, efficacy of prevention, and individual choice. In summary, actionability is a contextual assessment that will require careful consideration of how a lack of traditional FHx information might reframe our understanding of it.

\section{CONCLUSION}

At this time, the role of genomic analysis is most certainly an imperfect substitute for FHx. However, traditional FHx has significant limitations, too, in that it is the exception rather than the norm wherein FHx dramatically changes the approach to clinical care for most individuals. However, those cases where FHx does have utility are significant and such information can be life-saving. Therefore, mitigating lack of access to $\mathrm{FHx}$ through the thoughtful provision of targeted, highly selected genomic information could represent a valuable application of this newly emerging technology to the adopted population. Prospective, targeted analysis of selected genes in this population should be explored to assess its benefits and risks, both of which might vary from the broad population with access to FHx. Its application may be appropriate for assessing risk in only a few conditions to begin with; however, as predictive capacity improves and as preventive modalities are eventually developed for an expanding set of genetic disorders, its benefits may expand. Studies of how such populations perceive and integrate targeted genomic information into their lives and their healthcare will be key to understanding the value of genetic information obtained outside the traditional context of known family health history.

\section{ACKNOWLEDGMENTS}

For T.M. and K.A.S., work on this manuscript was partially funded by the Advancing a Healthier Wisconsin Research and Education Program Fund, a component of the Advancing a Healthier Wisconsin endowment at the Medical College of Wisconsin. 


\section{DISCLOSURE}

The authors declare no conflict of interest.

\section{REFERENCES}

1. Guttmacher AE, Collins FS, Carmona RH. The family history-more important than ever. N Engl J Med 2004;351:2333-2336.

2. Claassen L, Henneman L, Janssens AC, et al. Using family history information to promote healthy lifestyles and prevent diseases: a discussion of the evidence. BMC Public Health 2010;10:248.

3. Berg AO, Baird MA, Botkin JR, et al. National Institutes of Health State-ofthe-Science Conference Statement: Family History and Improving Health. Ann Intern Med 2009;151:872-877.

4. Zöller B, Li X, Sundquist J, Sundquist K. Familial transmission of prostate, breast and colorectal cancer in adoptees is related to cancer in biological but not in adoptive parents: a nationwide family study. Eur J Cancer 2014;50:2319-2327.

5. United Nations. Child adoption: Trends and Policies. 2009. http://www.un.org/ esa/population/publications/adoption2010/child_adoption.pdf. Accessed 10 April 2015

6. Hill CM, Edwards M. Birth family health history: adopters' perspectives on learning about their child's health inheritance. Adopt Fostering 2009:33:45-53.

7. Vandivere S, Malm K, Radel L. Adoption USA: A Chartbook Based on the 2007 National Survey of Adoptive Parents. Washington, DC: US Department of Health and Human Service, Office of the Assistant Secretary for Planning and Evaluation, 2009.
8. Schulte JM, Maloney S, Aronson J, San Gabriel P, Zhou J, Saiman L. Evaluating acceptability and completeness of overseas immunization records of internationally adopted children. Pediatrics 2002;109:E22.

9. Jones VF; Committee On Early Childhood, Adoption, And Dependent Care. Comprehensive health evaluation of the newly adopted child. Pediatrics 2012;129:e214-e223.

10. Heald B, Edelman E, Eng C. Prospective comparison of family medical history with personal genome screening for risk assessment of common cancers. Eur $J$ Hum Genet 2012;20:547-551.

11. Agalliu I, Wang Z, Wang T, et al. Characterization of SNPs associated with prostate cancer in men of Ashkenazic descent from the set of GWAS identified SNPs: impact of cancer family history and cumulative SNP risk prediction. PLOS One 2013;8:e60083.

12. Evans JP, Berg JS, Olshan AF, Magnuson T, Rimer BK. We screen newborns, don't we?: realizing the promise of public health genomics. Genet Med 2013;15:332-334.

13. Prince $A E$, Berg JS, Evans JP, Jonas DE, Henderson G. Genomic screening of the general adult population: key concepts for assessing net benefit with systematic evidence reviews. Genet Med; e-pub ahead of print 18 September 2014.

14. Clinical Sequencing Exploratory Research Consortium. Projects. https://cserconsortium.org/projects.

15. Green R, Berg J, Grody W, et al. ACMG recommendations for reporting of incidental findings in clinical exome and genome sequencing. Genet Med 2013;15:565-574. 